\title{
High-redshift galaxies and the TP-AGB phase
}

\author{
Claudia Maraston \\ Oxford University, Denys Wilkinson Building, Keble Road, OX2 6RJ, Oxford, UK \\ email: maraston@astro.ox.ac.uk
}

\begin{abstract}
I discuss how galaxy fundamental parameters that are derived by comparing observational data with stellar population models, depend on the adopted models. The emphasis is on the treatment of the Thermally-Pulsing Asymptotic Giant Branch (TP-AGB) stellar phase, that is a source of major discrepancy between the various model renditions. In particular, I show that ages and stellar masses of galaxies decrease when models including an empiricallycalibrated TP-AGB phase are considered. Illustrative examples are provided for a wide redshift range. Constraints to galaxy formation models need revision on the light of these results.
\end{abstract}

Keywords. stars: AGB and post-AGB; galaxies: star clusters; galaxies: evolution; galaxies: fundamental parameters; galaxies: high-redshift

\section{Introduction}

The observed properties of the stellar populations of galaxies, such as ages, metallicities, masses, set meaningful constraints to galaxy formation models, since stellar evolution is independent of cosmological models. The properties are derived by comparing the observed spectro-photometry with stellar population models. Obviously, the results are completely dependent on the prescriptions for stellar evolution adopted in such models, and the related uncertainties. As pointed out by Maraston (2005, hereafter M05), the treatment of the Thermally-Pulsing Asymptotic Giant Branch (TP-AGB) phase is the source of major discrepancy between the various models. In particular, the M05 models have a higher contribution from this phase when compared to widely used models like those by Bruzual \& Charlot (2003, hereafter BC03), Fioc \& Rocca-Volmerange (1997, Pegáse), Vazques \& Leitherer (2005, Starburst99). As a consequence, the M05 models are redder at the ages when the contribution of the TP-AGB phase is important, i.e. between 0.2 and 2 Gyr (see M05 for a detailed discussion).

These differences in the models were found to have an impact on the derived ages and metallicities of globular clusters (see e.g. Maraston et al. 2001)

For galaxies, this issue became particularly crucial after the Spitzer Space Telescope allowed the sampling of the rest-frame near-IR up to very high redshift. In fact, the TPAGB phase shines mostly in the near-IR. Moreover, high-redshift galaxies must contain TP-AGB stars, because at redshift around 3 the Universe itself is just a couple of billion years old, just the right age where the contribution of the TP-AGB phase is important. The use of different templates to interpret galaxy data will therefore have a non negligible effect on the galaxy properties that are derived by means these comparisons.

In the following I discuss age and mass determinations for galaxies at various redshifts, to conclude with a comment on the various models. 

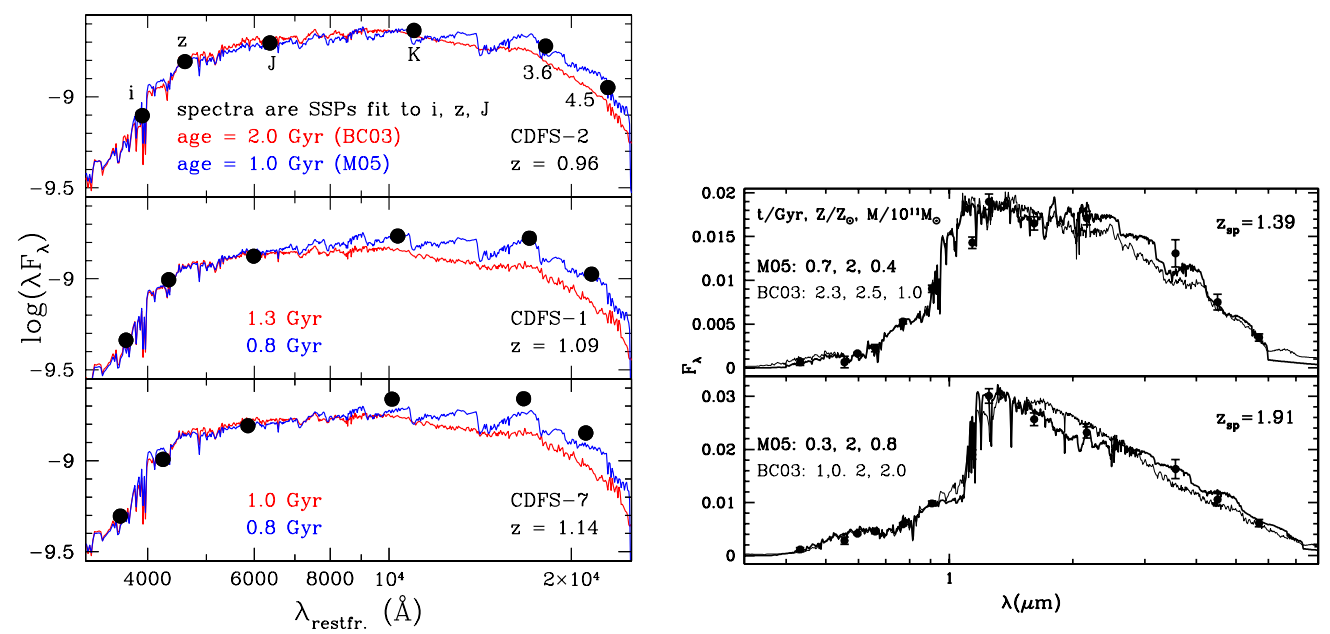

Figure 1. Left-hand panel: $z \sim 1$ early-type galaxies, the upper and lower fits refer to the M05 and BC03 models (from van der Wel et al. 2006). Right-hand panel: $z \sim 2$ passive early-type galaxies, thick and thin lines refer to M05 and BC03, respectively (from Maraston et al. 2006).

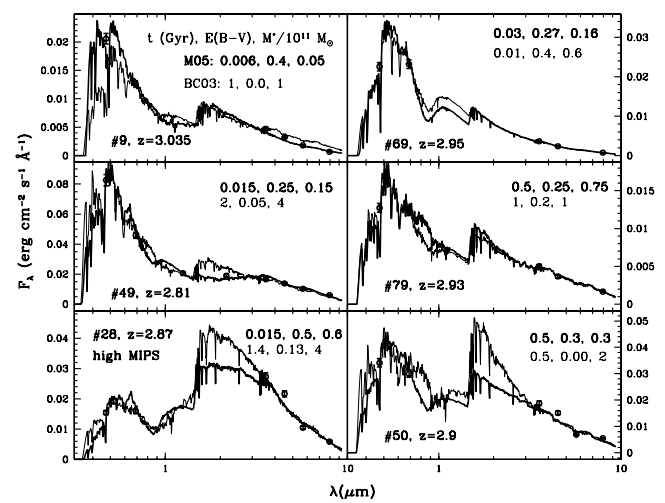

Figure 2. $z \sim 3$ Lyman-break galaxies, thick and thin lines refer to the M05 and BC03 templates, respectively. Ages, reddening $\mathrm{E}(\mathrm{B}-\mathrm{V})$ and masses are labelled in each plot. (In preparation, data from Rigoupolou et al. 2006).

\section{Effect of stellar population template as a function of redshift}

Figures 1 and 2 illustrate the same effect at various redshifts, namely how different properties such as ages and stellar masses, are derived for galaxies when different stellar population models are used. In each plot, the results obtained with the BC03 templates are compared with those referred to the M05 templates. In general, ages are smaller when the M05 templates are used, and consequently the inferred stellar masses are reduced. The size of the effect depends on the galaxy, and varies from a factor 2 to a factor 10 in the studied cases. Also, the best-fit of the observed spectral energy distributions generally improves.

The effect is due to the higher contribution of the TP-AGB phase in the M05 synthetic spectra, that makes these templates redder at a younger age ( $\sim 0.3 \mathrm{Gyr})$. In case of the BC03 models, instead, older ages, those at which RGB stars are developed ( $>1 \mathrm{Gyr}$ ), are required to match the near-IR. Older ages imply higher masses because low-mass stars are less luminous. These effects are described in detail in Maraston et al. (2006). 


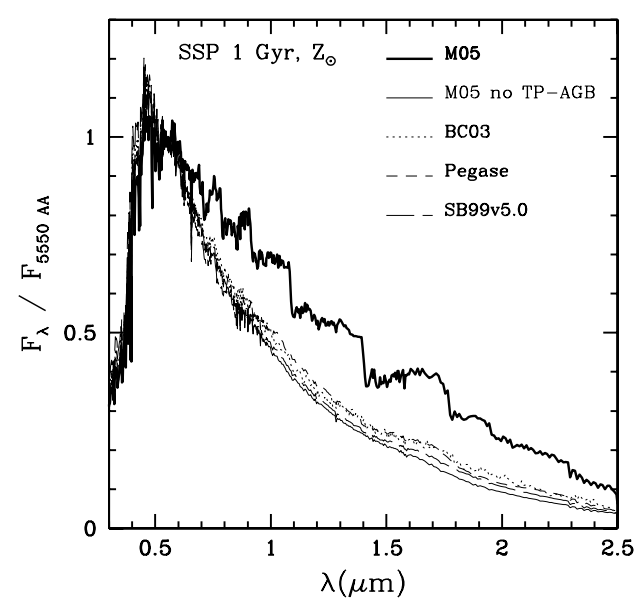

Figure 3. Synthetic SEDs of 1 Gyr Simple Stellar Population (SSP) with solar metallicity, according to different codes, including M05, BC03, Pegáse, SB99. A M05 model in which the TP-AGB phase is neglected is also shown.

It should be also noted that the above conclusions can be extended to cases in which templates by Pegáse or Starburst99 are used, because these models are very similar (due to almost identical input physics, see Figure 3 and M05). The stellar population models are explained in some detail in the next session.

\section{Why the TP-AGB matters? Energetics of Stellar Populations}

In a stellar population model, the integrated properties are computed by adding up the contributions by individual stars. The proper inclusion of all relevant stellar phases is therefore a critical issue. Notoriously, the most problematic stellar phase is the TP-AGB. The TP-AGB cannot be fully described in theory due to the occurrence of (unknown) mass loss, that affects luminosity, lifetime and effective temperature. However, the TPAGB should not be neglected since it is the dominant post-Main Sequence phase at ages $0.2 \lesssim t /$ Gyr $\lesssim 2$. In order to overcome to this difficulty, the models by Maraston resort to a semi-empirical description for this phase (Maraston 1998), that is based on the calibration of the TP-AGB energetics with data of Magellanic Clouds globular clusters (GCs, by Frogel, Mould \& Blanco 1990). The Magellanic Clouds are the only sites that contain GCs in the relevant age range, with ages and metallicities known independently, which allows a calibration to be performed. As shown in Figures 3 and 4, the treatment of the TP-AGB phase is the source of major discrepancy in the synthetic spectral energy distributions (SEDs) of different stellar population models. The M05 models stick out for having the highest near-IR flux and for displaying the molecular absorptions typical of Carbon-rich and Oxygen-rich stars. Both are the effects of the TPAGB phase. In Figure 4, I show a comparison between the M05 templates (thick lines) and the BC03 and Pegáse templates (thin lines) with the observed photometric SEDs of individual Magellanic Clouds GCs (one for sub-panel, data shown as symbols). The ages and metallicities that are used for the fitting template are independent determinations from the literature (see M05). It is clear from this comparison that only the M05 templates are able to match the observed SEDs of real stellar populations in the age range where the TP-AGB is important. In the other models the contribution of the TP-AGB phase is too low. 


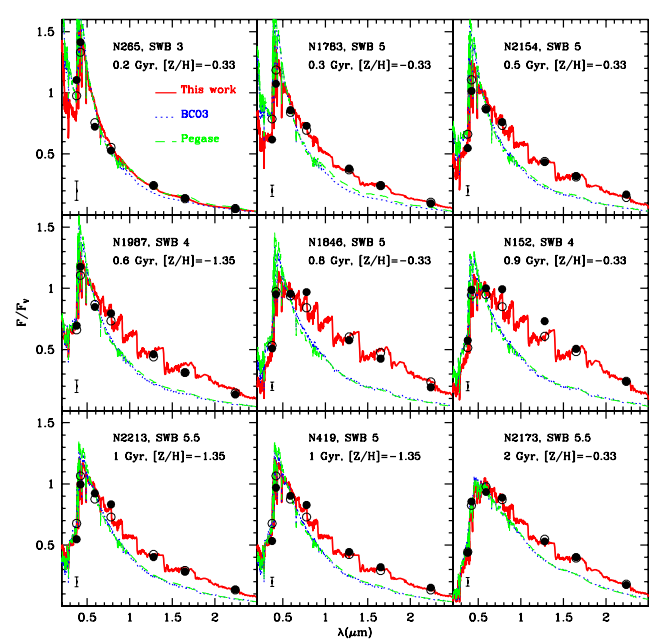

Figure 4. Comparison of stellar population models with the observed photometric SEDs of Magellanic Clouds GCs (filled symbols), of which IDs, ages and metallicities from the literature are labelled. The M05 models are the thick lines, the BC03 and Pegáse models the thin lines. From Maraston 2005.

\section{Conclusions}

I have discussed how a proper treatment of the TP-AGB phase in a stellar population model is required to describe the observed spectro-photometry of globular clusters as well as high-redshift galaxies. As widely known and also recalled at this meeting, stellar evolutionary models alone are not able to predict the whole extension of the TPAGB phase because unknown mass-loss shapes the extension, luminosity and effective temperature of the phase. The energetics and spectral types along the TP-AGB require calibration with data, as in the models by Maraston $(1998,2005)$. The use of these new models, besides improving the spectral energy distribution fitting, has a sizable effect on the properties that are derived for galaxies, in particular lower ages/formation redshifts, hence smaller masses are obtained. This impacts on our understanding of galaxy formation and evolution.

\section{Acknowledgements}

I thank the organizers for a wonderful meeting in a splendid location.

\section{References}

Bruzual, G., \& Charlot, S. 1993, MNRAS, 344, 1000, BC03

Fioc, G., \& Rocca-Volmerange, B. 1997 MNRAS, 326, 950, Pegase

Frogel, J.A., Mould, J., \& Blanco, V.M. 1990, ApJ, 352, 96

Maraston, C. 1998, MNRAS, 300, 872

Maraston, C. 2004 in Multiwavelength mapping of galaxy formation and evolution, eds. A. Renzini \& R. Bender, Springer

Maraston, C. 2005, MNRAS, 362, 799, M05

Maraston, C., et al. 2001, A\&A A, 652, 85

Maraston, C., et al. 2006, ApJ, 652, 85

Rigoupolou, D. et al. 2006, ApJ, 648, 81

van der Wel, A. et al. 2006, ApJ, 652, 97

Vázques, G.A., \& Leitherer, C., 2005, ApJ, 621, 695, Starburst99 


\section{Discussion}

SETH: The TP-AGB is a rare phase, what mass of cluster is needed for stochastic effects to no longer be important?.

Maraston: Among the Magellanic Clouds GCs of intermediate ages, those relevant to the development of the TP-AGB phase, one can minimize the stochastical fluctuations by grouping clusters with similar ages (see Maraston 98;05). These Magellanic Cloud clusters have masses $\sim 10^{4}-10^{5} M_{\odot}$. Above this value, the stochastical fluctuations are not important, as we show for example fo rhte star clusters in the merger remnant galaxy NGC7257, that have masses $\sim 10^{6} M_{\odot}$.

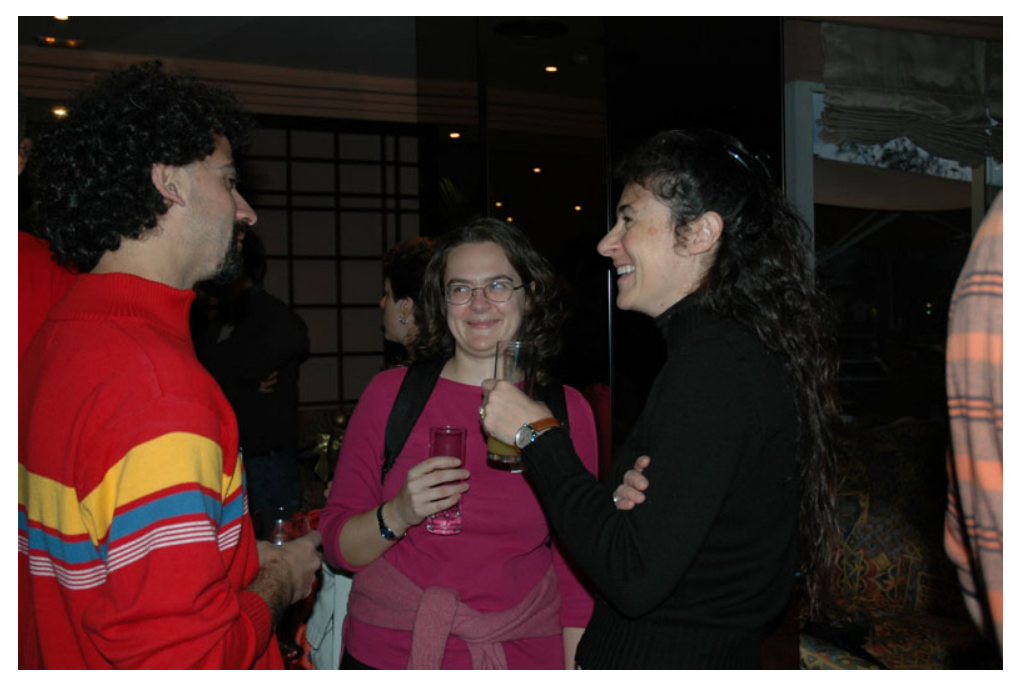

Lorenzo Morelli, Alessia Moretti and Rosaria Tantalo (f.l.t.r.).

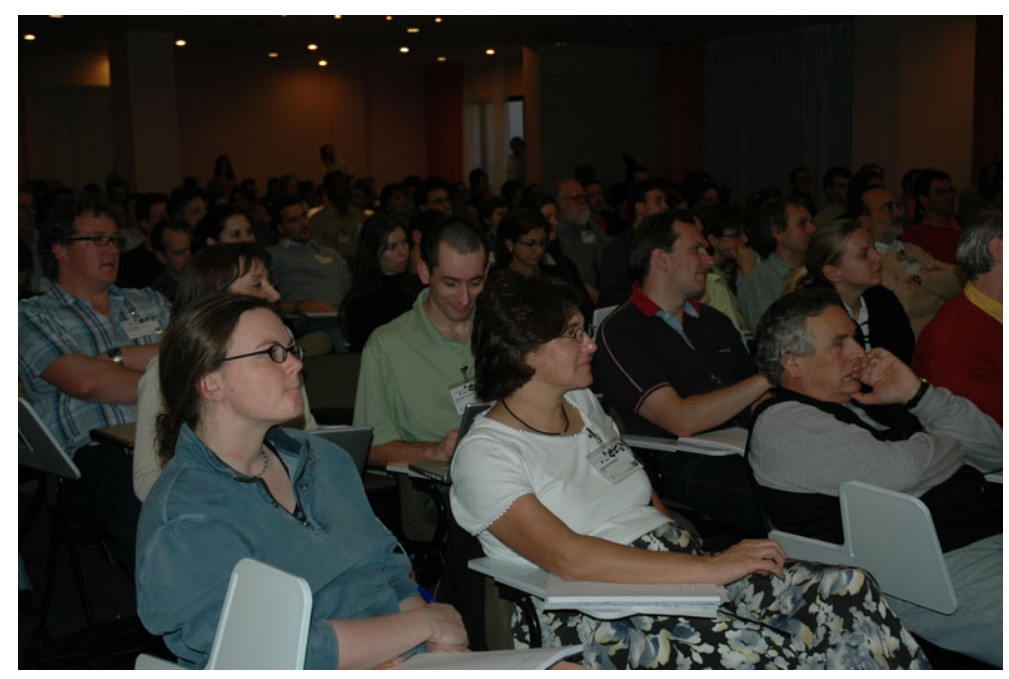

Front row (f.l.t.r.): Eline Tolstoy, Margaret Meixner and Alvio Renzini. 